\title{
The Effect of the Magnetic Field to the Microstructure and Sensitivity of $\mathrm{Cu} / \mathrm{Ni}$ Film
}

\author{
Azmi Khusnani ${ }^{1}$, Moh. Toifur ${ }^{1, *}$, Guntur Maruto ${ }^{2}$, Yudhiakto Pramudya ${ }^{1}$ \\ ${ }^{1}$ Department of Physics, Ahmad Dahlan University, Indonesia \\ ${ }^{2}$ Department of Physics, Gadjah Mada University, Sekip Utara Bulaksumur, Indonesia
}

Received July 25, 2019; Revised October 3, 2019; Accepted December 10, 2019

Copyright $\subseteq 2019$ by authors, all rights reserved. Authors agree that this article remains permanently open access under the terms of the Creative Commons Attribution License 4.0 International License

\begin{abstract}
Cu} / \mathrm{Ni}$ thin film has been made by electroplating method assisted by magnetic fields. The solution consists of a mixture of $\mathrm{NiSO}_{4}(260 \mathrm{~g} / \mathrm{L}), \mathrm{NiCl}_{2}$ $(60 \mathrm{~g} / \mathrm{L})$ and $\mathrm{H}_{3} \mathrm{BO}_{3}(40 \mathrm{~g} / \mathrm{L})$. The magnetic field varied in the range of 0-200 gauss in a direction perpendicular to the electric field. Plating was carried out at a voltage of 1.5 volts, the electrolyte temperature is $60{ }^{\circ} \mathrm{C}$ during $5 \mathrm{~s}$. The results indicate that the $\mathrm{Ni}$ film has been formed on the surface of the $\mathrm{Cu}$ plate. Testing of sheet resistivity shows that by coating $\mathrm{Ni}$ on $\mathrm{Cu}$ substrate the resistivity sheet of $\mathrm{Cu} / \mathrm{Ni}$ increases. The increase in resistivity is proportional to the magnitude of the magnetic field. Investigation on the Ni morphology shows that the larger the magnetic field the smaller the grain size so that the composition of $\mathrm{Ni}$ particles becomes more tightly packed. Whereas, in sensor sensitivity, all samples showed their role as low-temperature sensors. The sensitivity decreases along with the magnitude of the magnetic field given.
\end{abstract}

Keywords $\mathrm{Cu} / \mathrm{Ni}$ Film, Low-temperature, Magnetic Field, Microstructure, Sensitivity

\section{Introduction}

Preservation is a method to maintain the quality of the material so that it can be reused for a long time [1]. The method of preserving human bodies that have been used is preservation using the cryonics method [2]. Cryonics is a preservation method at liquid nitrogen using cryoprotectant substances [3-5]. In liquid nitrogen media, preserved cells will remain in good condition and can later be reused [6]. If at the preservation site liquid nitrogen experience evaporation at, temperature rise up to critical temperature about $-100^{\circ} \mathrm{C}$, and damage to preserved organs. Therefore, to prevent this situation, sensor technology is needed to monitor the temperature of liquid nitrogen continuously.

One sensor technology that can be used for low-temperature detectors is RTD-F (Film-Resistance Temperature Detector) [7]. Toifur et al. have developed a low-temperature sensor made from $\mathrm{Cu} / \mathrm{Ni}$ thin film prepared by the electroplating method [8]. From the analysis of the microstructure of the material on the XRD diffractogram, it was found that the crystal structure was formed in the film. In addition, the layers contain 3 phases, namely $\mathrm{Cu}, \mathrm{Ni}$, and $\mathrm{NiO}$. However, from the SEM photos, the surface size of Ni particles has not been homogeneous.

The surface morphology of the non-homogeneous deposit can be repaired by doing plating which is assisted by a magnetic field during the deposition process [9-13]. This can happen because, when the deposition process takes place, the current flows from the cathode to the anode. If the position of the magnetic field is perpendicular to the electric force, there will be a Lorentz force whose magnitude competes with the electric force [14]. With the two positions of forces that are perpendicular to each other, the growth of deposit at the cathode surface will increase, which is because of the deposition process which is influenced by the magnetic field, will accelerate mass transport $[15,16]$. In the electrodeposition process, the magnetic field greatly affects the yield of the resulting deposit layer, the magnetic field given will affect to the Lorentz force generated [11].

In this study, $\mathrm{Ni}$ plating on $\mathrm{Cu}$ substrates will be carried out with the help of a magnetic field perpendicular to the electric field. The magnitude of the magnetic field varies from 0 to 200 gauss and its effect on surface fineness will be observed. Due to this material that will be used as a low-temperature sensor, after obtaining the most suitable field to produce a layer with a homogeneous microstructure, the sample will be used to measure the temperature of liquid nitrogen from $0{ }^{\circ} \mathrm{C}$ to $-170{ }^{\circ} \mathrm{C}$..

\section{Materials and Methods}

The process of making $\mathrm{Cu} / \mathrm{Ni}$ layers begins by 
providing a $10 \times 1.3 \mathrm{~cm}^{2}$ copper plate. This plate will be installed as a cathode. Similarly, the preparation for nickel plates was installed at the anode. The materials used for preparing electrolyte solution are $\mathrm{NiSO}_{4}, \mathrm{NiCl}_{2}, \mathrm{H}_{3} \mathrm{BO}_{3}$ with the weight $260 \mathrm{~g}, 60 \mathrm{~g}, 40 \mathrm{~g}$ respectively. These materials then were diluted in $1000 \mathrm{~mL}$ of distilled water. The ingredients are mixed and then stirred using a magnetic stirrer for 6 hours.

Experiments are carried out by assembling tools such as Fig. 1. The deposition process is carried out at a voltage of $1.5 \mathrm{~V}, 4 \mathrm{~cm}$ electrode distance, 5 seconds deposition time, and $60{ }^{\circ} \mathrm{C}$ of bath temperature. To generate a 200 gauss magnetic field, it used a pair of coils made of copper wire with a diameter of $0.2 \mathrm{~mm}$, a number of 1500 coil turns. By regulating the input voltage, the magnetic field can be varied from 0 to 200 gauss.

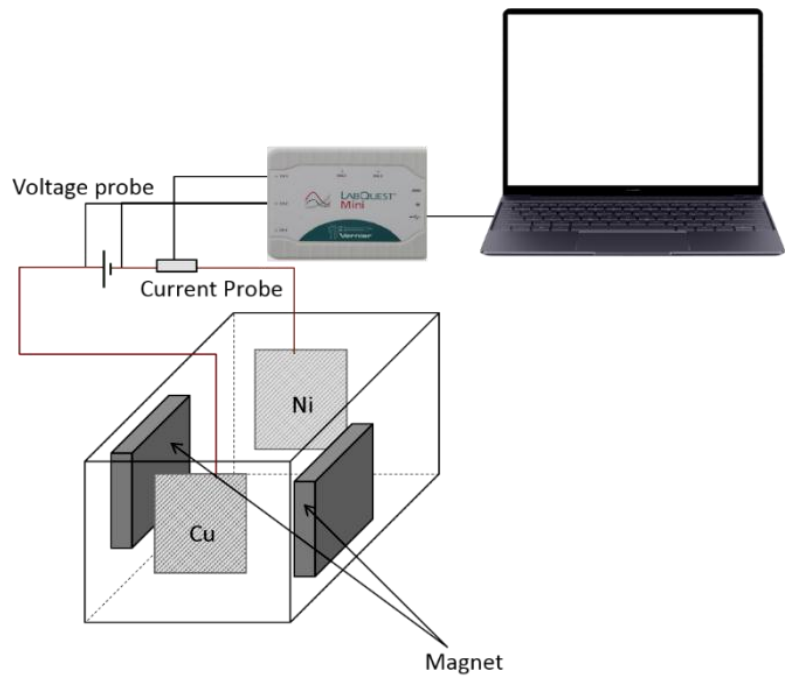

Figure 1. Ni deposition devices assisted the magnetic field

Characterization of $\mathrm{Cu} / \mathrm{Ni}$ layers was carried out by looking the microstructure of samples with several instruments that are SEM for surface morphology, EDS for elemental tracing, and XRD for detecting the formation of crystalline structure. From the XRD pattern it can obtain the intensity, grain size, and d-spacing.
While the characterization of sheet resistivity is done using a four-point probe (as pointed at Fig. 2) at room temperature. This test is carried out by varying the current (I) to produce $V$ and data processing is done by linear regression of $\left(V_{i}, I_{i}\right)$. The value of Rs is obtained from the slope of graph $(a)$ with the equation:

$$
R_{s}=\frac{\pi}{\ln 2} \frac{V}{I}=\frac{\pi}{\ln 2} a
$$

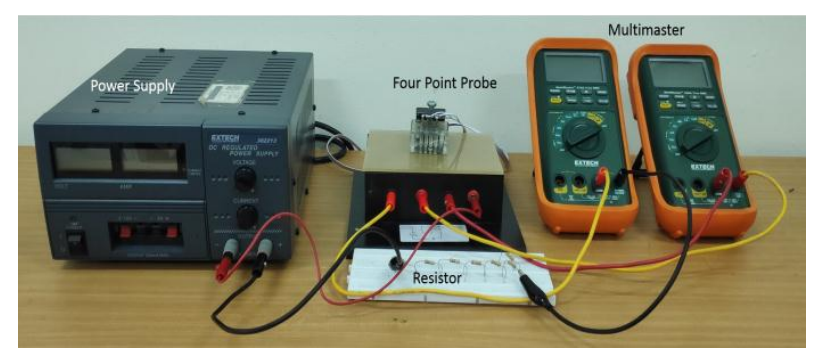

Figure 2. Four point probe

Testing performance sensor is done in liquid nitrogen at a temperature of 0 to $-200{ }^{\circ} \mathrm{C}$ by inserting slowly the sensor into a flask at the rate of $0.2 \mathrm{~cm} / \mathrm{s}$ (as pointed at Fig. 3 ). The flask contains 2 liters of liquid nitrogen with different temperature between the top and the bottom. Output data was displayed in the form of the output voltage at various temperatures. This voltage varies depending on the temperature of the medium. Voltage variations occur due to changes in the value of sensor resistance. The temperature of the medium varies according to the depth of the flask. the more the temperature gets lower. Voltage $V$ and temperature $T$ have a relationship of

$$
V=a T^{2}+b T+c
$$

The strength of the relationship between the two variables $V$ and $T$ can be seen through the determination index $R^{2}$. Furthermore, each characteristic in the $2^{\text {nd }}$ order polynomial equation can be investigated from the coefficient values $a, b, c$. The $a$ shows the graph of the level of curvature on the curve, The $b$ shows the level of the slope of the graph, and the $c$ as an intercept. 


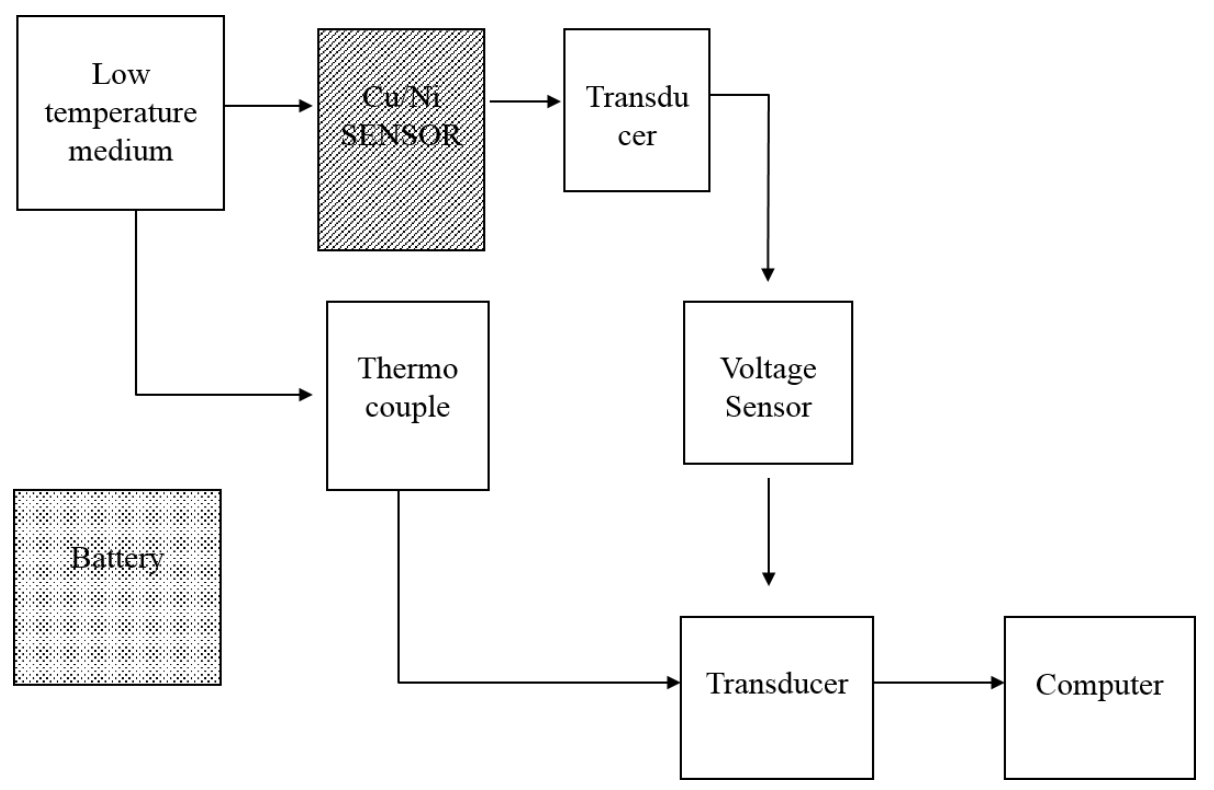

Figure 3. Schematic diagram for producing and determinating sensitivity of $\mathrm{Cu} / \mathrm{Ni}$ sensor

\section{Results and Discussion}

Based on Fig. 4, it is known that each sample has a different surface morphology. In samples with $B=50$ gauss, Ni grains are larger than Ni grains in samples with $B=0$ gauss. By comparing the overall $\mathrm{Ni}$ layer morphology it is known that the increase in the magnetic field produces $\mathrm{Ni}$ deposits that have smaller granules. However, it also appears that the grain size of the $\mathrm{Ni}$ particle in one sample is not homogeneous. Here, it is seen that the use of a magnetic field perpendicular to the direction of deposition leads to the emergence of the Lorentz force. The presence of this Lorentz force causes the travel of $\mathrm{Ni}$ ions to the $\mathrm{Cu}$ cathode to have a helical path [15]. The farther the distance the more deviated. As a result, the distribution of $\mathrm{Ni}$ ions to the $\mathrm{Cu}$ substrate is not uniform. In this condition, the $\mathrm{Cu}$ substrate in one end receives more $\mathrm{Ni}$ ion files compared to the other end.

This is likely due to the influence of Lorentz force so $\mathrm{Ni}$ 's ionic films that should move straight from the anode to the cathode to be deviate so that some $\mathrm{Ni}$ ions do not collide the substrate. Another possibility is the presence of magnetic damping forces arising from variations in the Lorentz force that interacts with the magnetic field. The Lorentz force varies because the surface of the substrate is uneven so that an induced current is produced. This induction current when interacting with a magnetic field will produce the damping force on $\mathrm{Ni}$ ion [18]. As large as the magnetic field the greater the damping force so that the mass of $\mathrm{Ni}$ formed at the cathode becomes less than the $\mathrm{Ni}$ mass produced in plating without using a magnetic field.

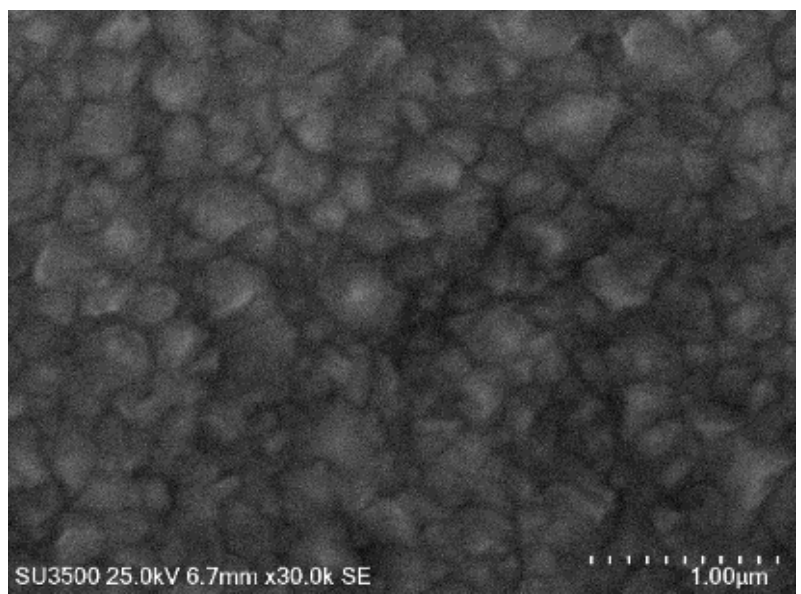

(a)

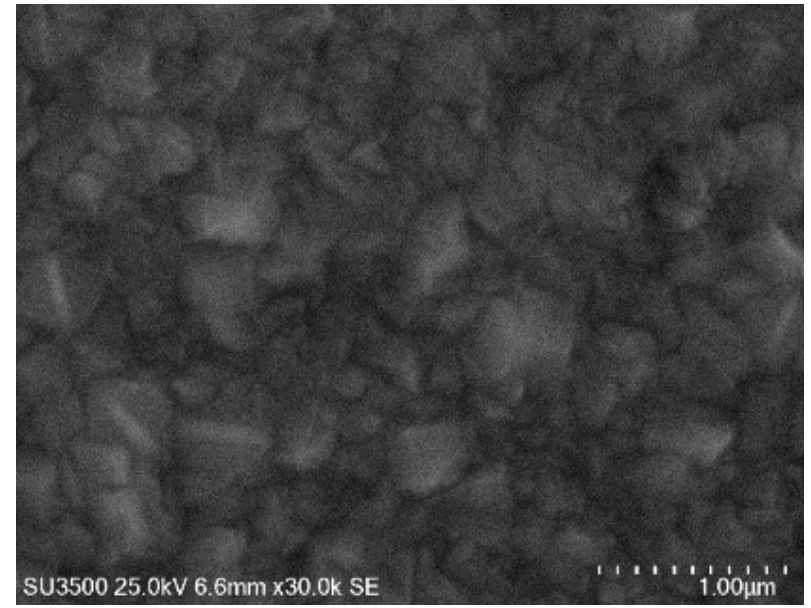

(b) 


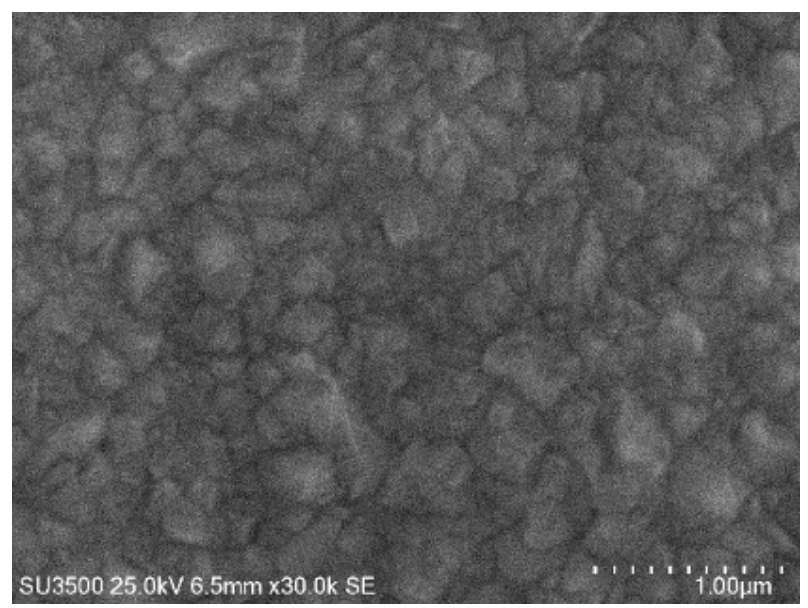

(c)

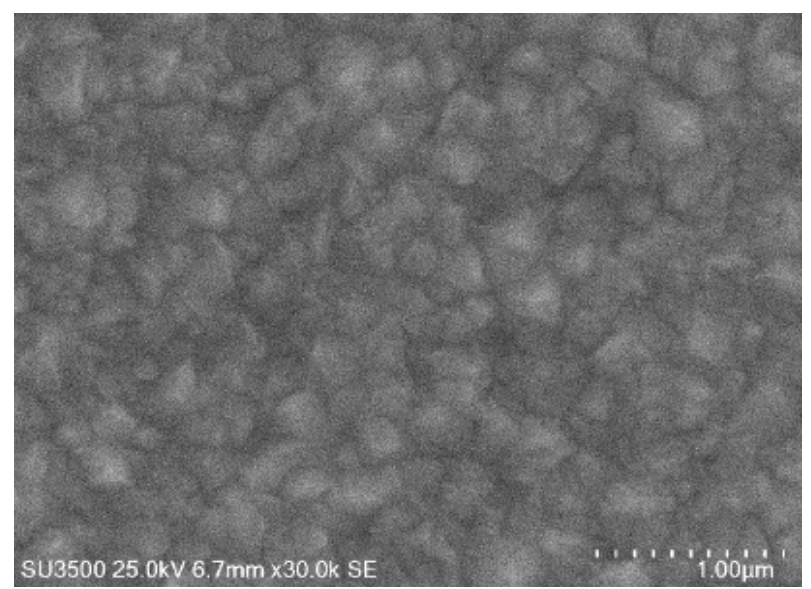

(d)

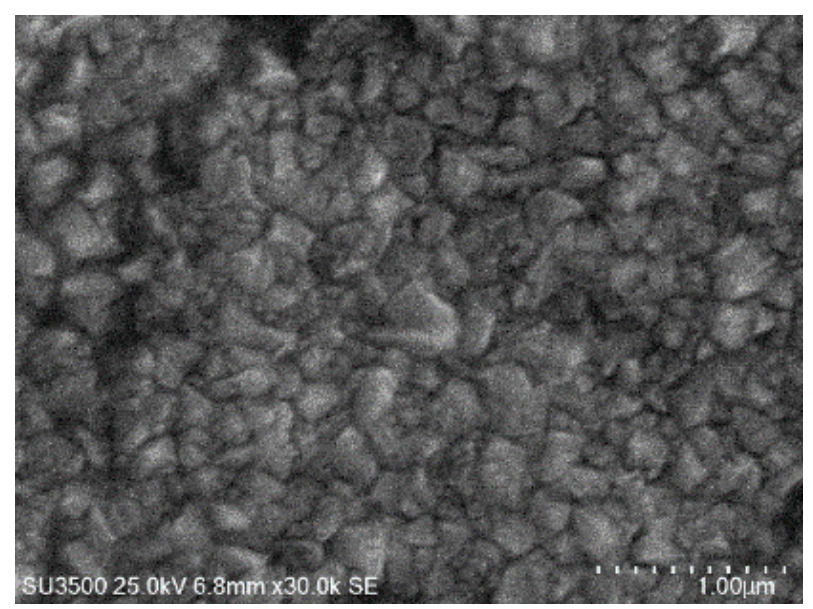

(e)

Figure 4. $\mathrm{EM}$ photos of $\mathrm{Cu} / \mathrm{Ni}$ surfaces in variation magnetic field with a) 0 gauss, b) 50 gauss, c) 100 gauss, d) 150 gauss and e) 200 gauss

The rate of $\mathrm{Ni}$ growth on $\mathrm{Cu}$ surface in the deposition process can be seen from the electric current as shown in Fig. 5 There is a tendency for the use of magnetic field that is an increase in electric current during the deposition process. At $B=100$ gauss the in-crease in deposition current is highest compared to other $B$. For all $B$ the current starts to stabilize for all $B$ in approximately $1.5 \mathrm{~s}$. Unlike the deposition without a magnetic field, the current is stable.

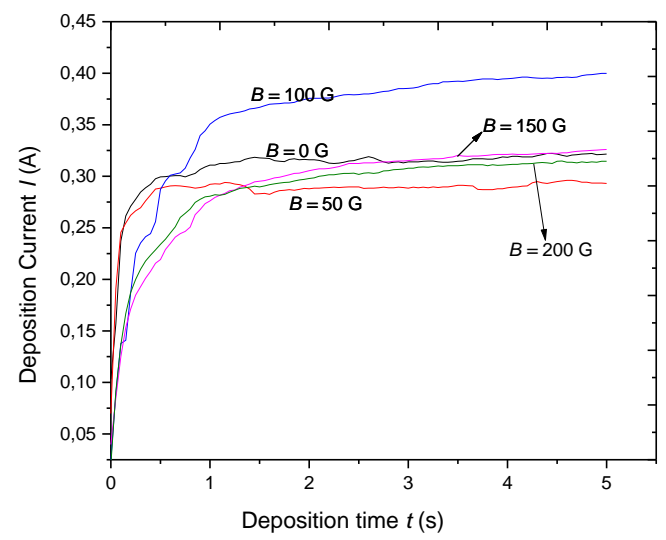

Figure 5. Deposition current at various magnetic field

Therefore, it is according to what was said by Ebadi [13] that the use of magnetic fields can increase the Ni mass transport process towards $\mathrm{Cu}$. For $B=100$ gauss the highest increase in deposition current, which means acceleration of deposition.

In Fig. 6 an X-ray diffraction pattern is displayed for identifying the microstructure parameters of the material. Based on the Fig., it is known that all of the peaks belonging to $\mathrm{Cu}$ with the dominant peak around the diffraction angle of $44^{\circ}$ in the direction of [111]. The picture does not appear to be the Ni peak. Therefore it is estimated that $\mathrm{Ni}$ deposition for $5 \mathrm{~s}$ has not produced a Ni-crystal structure. However, from SEM photos as shown in Fig. 4 and Fig. 5, Ni layers have been formed.

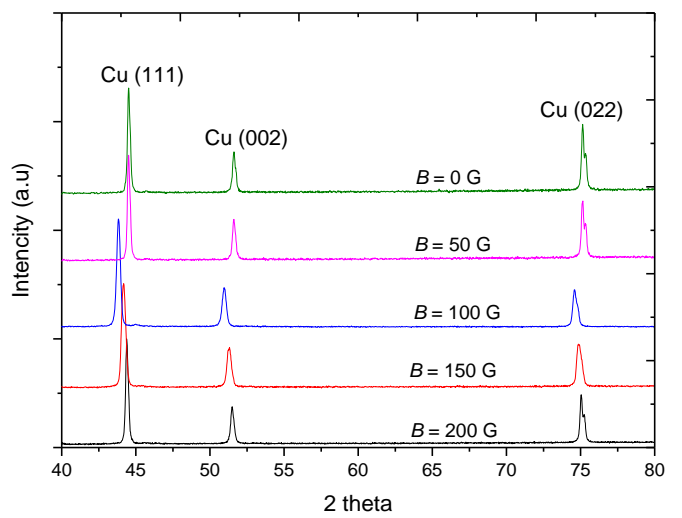

Figure 6. X-ray diffractogram of $\mathrm{Cu} / \mathrm{Ni}$ for variation $B$

From the electrical properties of $\mathrm{Ni}$, deposition $\mathrm{Ni}$ on $\mathrm{Cu}$ causes an increase of the sheet resistivity, where the sheet resistivity of $\mathrm{Cu}$ before plating is $(1.172 \pm 0.03)$ $\mathrm{x} 10-3 \Omega / \mathrm{sq}$, and after plating to form $\mathrm{Cu} / \mathrm{Ni}$ it sheet resistivity increases as shown in Fig. 7. 


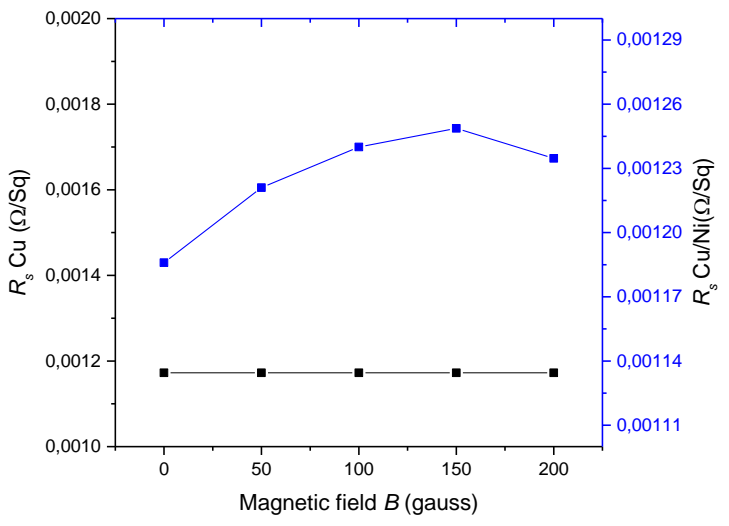

Figure 7. Graph of the relationship between $R s$ for $\mathrm{Cu}$ and for $\mathrm{Cu} / \mathrm{Ni}$ in variation $B$

Furthermore, all the samples have known resistivity, then all samples are tested for performance as low temperature sensors. One of the sensor performance parameters is expressed in the form of sensor sensitivity level in response to medium temperature. In Fig. 8 the sensor output voltage is displayed against the medium tem-perature from $-170{ }^{\circ} \mathrm{C}$ to $0{ }^{\circ} \mathrm{C}$. From the picture it appears all data $\left(V_{i}, T_{i}\right)$ have a tendency to form polynomials. Therefore the data $\left(V_{i}, T_{i}\right)$ is diffused according to order polynomial 2 . The results are given in Table 1

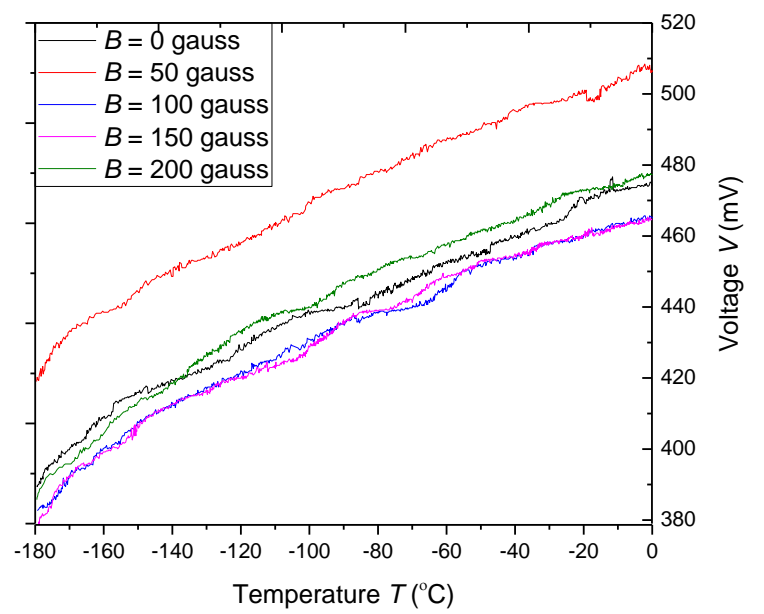

Figure 8. Graph of the relationship between voltage and temperature liquid nitrogen

Table 1. Relationship between sensor voltage and temperature

\begin{tabular}{|c|c|c|}
\hline$B$ (gauss) & $V=a T^{2}+b T+c$ & $R^{2}$ \\
\hline 0 & $V=-6.650 \mathrm{E}-7 T^{2}+0.317 \mathrm{E}-3 T+0.475$ & 0.99 \\
\hline 50 & $V=-1.080 \mathrm{E}-6 T^{2}+0.261 \mathrm{E}-3 T+0.506$ & 0.99 \\
\hline 100 & $V=-1.160 \mathrm{E}-6 T^{2}+0.229 \mathrm{E}-3 T+0.465$ & 0.99 \\
\hline 150 & $V=-1.370 \mathrm{E}-6 T^{2}+0.206 \mathrm{E}-3 T+0.465$ & 0.99 \\
\hline 200 & $V=-1.560 \mathrm{E}-6 T^{2}+0.192 \mathrm{E}-3 T+0.476$ & 0.99 \\
\hline
\end{tabular}

The sensitivity level is taken at the coefficient $b$. Samples that have the largest $\mathrm{b}$ are most sensitive in response to temperature.it is known that all the $\mathrm{Cu} / \mathrm{Ni}$ thin films have behaved as a low tem-perature sensor. On the other hand from the $R^{2}$ that is 0.99 all samples have a good relationship between $V$ and $T$. The value of sensitivity is taken based on $T$ coefficient.

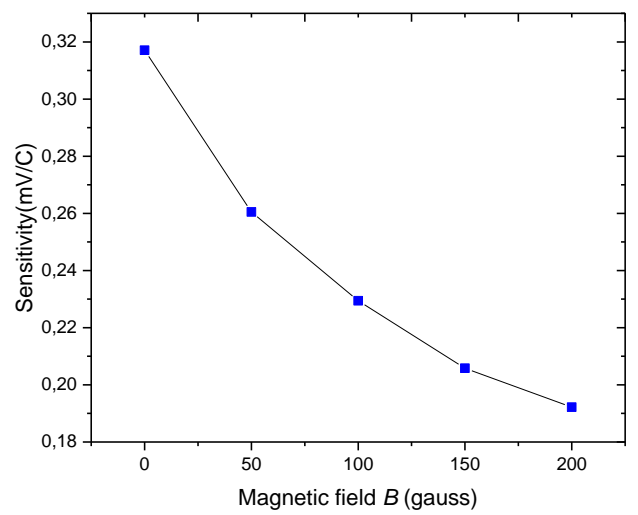

Figure 9. Effect of magnetic field on the sensitivity of the sensor

Sensitivity is a change in temperature that can be responded to as the change of a sensor voltage. Sensitivity corresponds to the coefficient $b$ in equation (2). In Fig. 9 a graph of sensor sensitivity is displayed on various deposition fields $B$. The highest sensitivity $(0.317 \pm 0.006)$ $\mathrm{mV} /{ }^{\circ} \mathrm{C}$ corresponds to a magnetic field of 0 gauss and the lowest sensitivity $(0.192 \pm 0.004) \mathrm{mV}^{\circ}{ }^{\circ} \mathrm{C}$ in accordance with the magnetic field of 200 gauss. By observing Fig. 8 and Fig. 9, it can be obtained that the resistivity of the $\mathrm{Cu} / \mathrm{Ni}$ thin layer is inversely proportional to the sensitivity.

\section{Conclusions}

Based on all of the data that have been analyzed, we conclude that electroplating by using a magnetic field in the direction perpendicular to the electric field can emerge the Lorentz force. This force will shift the Ni ions leading to $\mathrm{Cu}$ cathode. Therefore, the distribution of $\mathrm{Ni}$ in $\mathrm{Cu}$ plate from the end to the end one is inhomogeneous. But, the using of the magnetic field has been able to improve the microscopic condition of the Ni layer, which is the greater the $B$, the smaller the grain size. Thiswill make sheet resistivity get the increase. The $5 \mathrm{~s}$ time deposition has not produced the crystalline structure on $\mathrm{Ni}$ film. From the study concerning the sensitivity of $\mathrm{Cu} / \mathrm{Ni}$ sensor it is found that the increasing magnetic field leads to the decrease in the sensor's sensitivity.

\section{Acknowledgements}

This research is a part of main research according to an 
improvement of the quality of low temperature sensor through synthesis of $\mathrm{Cu} / \mathrm{Ni}$ thin film assisted magnetic fields. The researcher expresses his deepest gratitude to the Ministry of Research, Technology and Higher Education of the Republic of Indonesia which has provided research funding through the 2019 Master Thesis Research Grant (PTM) Scheme with contract No. PTM-020/SKPP.TT/ LPPM UAD/III/2019.

\section{REFERENCES}

[1] J. Y. Balta, M. Cronin, J. F. Cryan, S. M. O'mahony, Human Preservation Techniques in Anatomy: A 21st-Century Medical Education Perspective, Clinical Anatomy, Vol. 28, No.6, 725-734, 2015.

[2] A. d. Wolf, Identification Validation and Implementation of View Cryonics Technologies, Germany: Advanced Neural Biosciences, Inc, 2014.

[3] A. Pandhi, Chilling Legalities of Cryopreservation. International Journal of Socio-Legal Analysis and Rural Development, II No. 3, 96-97, 2015.

[4] M. S. Bhattacharya, A Review on Cryoprotectant and Its Modern Implication in Cryonics, Asian Journal of Pharmaceutics (AJP): Free full text articles from Asian J Pharm, Vol. 10 No. 3, 154-159, 2016.

[5] H. Thomson, The Big Freeze, New Scientist, Vol. 231 No. 3080, 26-31, 2016.

[6] R. Cron, Is Cryonics an Ethical Means of Life Extension? University of Exeter, 4, 2014.

[7] M. Lebioda, J. Rymaszewski, Dynamic Properties of Cryonics Temperature Sensors, Przegląd Elektrotechniczny, Vol. 2 No. 1, 225, 2015.

[8] M. Toifur, Y. Yuningsih, A. Khusnani, Microstructure, Thickness and Sheet Resistivity of $\mathrm{Cu} / \mathrm{Ni}$ Thin Film Produced by Electroplating Technique on The Variation of Electrolyte Temperature, Journal of Physics: Conference Series, 2-6, 2018.

[9] Y. Yu, Z. Song, H. Ge, G. Wei, L. Jiang, Effects of Magnetic Fields on the Electrodeposition Process of Cobalt, International Journal of Electrochemical science, No. 10, 4812-4813, 2015.

[10] L. M. Monzon, J. M. Coey, Magneticfields in Electrochemistry: The Kelvin force, A Mini-Review, Electrochemistry Communications, 42-43, 2014.

[11] L. T. Xia, G. Y. Wei, M. G. Li, H. F. Guo, Y. Fu, H. Dettinger, Preparation of Co-Pt-P Thin Films by Magnetic Electrodeposition, Material Research Innovations, Vol. 5 No. 18, 386-389, 2014.

[12] Sudibyo, M. B. How, N. Aziz, Influences of Magnetic Field on The Fractal Morphology in Copper Electrodeposition, Mineral Processing and Technology International Conference, IOP Conference Series, 4-6, 2017.

[13] M. Ebadi, W. J. Basirun, Y. Alias, Influence of Magnetic
Field on The Electrodeposition of Ni-Co Alloy, Journal Chemical Science, Vol. 2 No. 122, 279-280, 2010.

[14] Y. D. Yu, Z. L. Song, H. L. Ge, G. Y. Wei, Influence of Magnetic Fields on Cobalt Electrodeposition. Surface Engineering, Vol. 2 No. 30, 83-85, 2014.

[15] Y. D. Yu, Y. Cao, M. G. Li, G. Y. Wei, H. Dettinger, Magnetic Electrodeposition of $\mathrm{ZnNi}$ Alloy Films, Material Research Innovations, Vol. 4 No. 18, 314-317, 2014.

[16] Y. D. Yu, H. F. Guo, J. W. Lou, H. L. Ge, G. Y. Wei, CoPtW Thin Films Prepared by Magnetic Electroplating, Material Research Innovations, Vol. 3 No.16, 183, 2013.

[17] J. M. D. Coey, G. Hinds, Magnetic Electrodeposition, Journal of Alloys and Compounds, No. 326, 238-245, 2001. 